\title{
Robust Programmable-Rate Wireless Graphical Correspondence
}

\author{
Lajos Hanzo, ANdy H. Yuen \\ Dept. of Electr. and Comp. Sc., University of Southampton \\ Mountbatten Builaing, 4003, SO17 1BJ, UK
}

\begin{abstract}
An adaptive fixed-length differential chain coding (FL-DCC) scheme is proposed for the transmission of line graphics, which canbe re-configured as a lower rate, lower resolution or higher rate, higher resolution source codec. Compared to Differential Chain Coding (DCC), the proposed FL-DCC codec has a lower coding rate in both of its lower- and higher-rate operating modes, while maintaining a similar subjective graphical quality.The dual-rate re-configurable FL-DCC codec is embedded in a voice, video multimedia communicator system, which allocates the FL-DCC graphics packets to idle speech packels with the aid of the Packet Reservation Multiple Access (PRMA) multiplexer. In a bandwidth of $200 \mathrm{kHz}$, which is characteristic of the Pan-European GSM system, nine speech, video and graphics multimedia users can be accommodated using bandwidth efficient 16-level Quadrature Amplitude Modulation (I6QAM) over benign microcellular channels. Under less favourable propagation conditions the more robust, but less bandwidth efficient 4QAM mode of operation can be invoked. In the diversity-aided and automatic repeat request (ARQ) assisted 16QAM mode of operation the multimedia user bandwidth becomes $22.2 \mathrm{kHz}$ and the minimum required channel signal to noise ratio (SNR) over AWGN and Rayleigh channels is about 11 and $15 \mathrm{~dB}$, respectively. The most salient system features are summarised in Table 2.
\end{abstract}

\section{INTRODUCTION}

Telewriting is a multimedia telecommunication service enabling the bandwidth-efficient transmission of handwritten text and line graphics through fixed and wireless communication networks $[2,4]$. Differential chain coding (DCC) has been successfully used for graphical communications over E-mail networks or teletext systems [1], where bit rate economy is achieved by exploiting the correlation between successive vectors. A plethora of further excellent treatises were contributed to the literature of chain coding by R. Prasad and his colleagues from Delft University [1,5].

In this contribution an intelligent re-configurable graphical communications scheme is contrived, which is embedded in a voice, video multimedia system context. In the proposed fixed-length differential chain coding (FL-DCC) scheme the number of bits used to quantise the differential vectors of the so-called coding ring is dynamically adjusted under system's control, in order to match the prevailing bitrate, graphical quality and/or channel quality constraints. Our efforts are in line with the European Research in Advanced Communications Equipment (RACE) project's Advanced Communications Technologies and Services (ACTS) programme [6] to define a flexible third generation standard, backwardly compatible with second generation systems, while providing more advanced future features. The adaptivity of the transceiver can be exploited in numerous ways on a more static or dynamic basis, but these algorithms form part of the higher-level functions of the classic sevenlayer architechture. This contribution will concentrate on the physical layer functions, establishing the principle and documenting the expected performance of the adaptive graphical transceiver, while leaving the issues of re-configuration algorithms for future studies. Although during low bitrate operation our transceiver uses lossy quantisation, we will demonstrate that in case of small coding rings near-unimpaired subjective quality is maintained.

The paper is structured as follows. Section 2 highlights the system's architecture, leading to section 3, which describes the proposed re-configurable graphical source codec. The codec's robustness against channel errors is increased using a technique described in section 4. Transmission issues, including error control, modulation and packetisation aspects are discussed in section 5. Section 6 is devoted to issues related to multiplexing graphical source signals with voice and video, before reporting on the system's performance in section 7. Our conclusions are offered in section 8.

\section{SYSTEM ARCHITECTURE}

The proposed system's architecture is shown in Fig. 1, where the voice, video and graphics source encoders' bit streams are mapped in two sensitivity classes and sensitivity-matched binary Bose-Chaudhuri-Hocquenghem (BCH) forward error correction (FEC) coded [11]. Then both the more vulnerable Class One and the more robust 


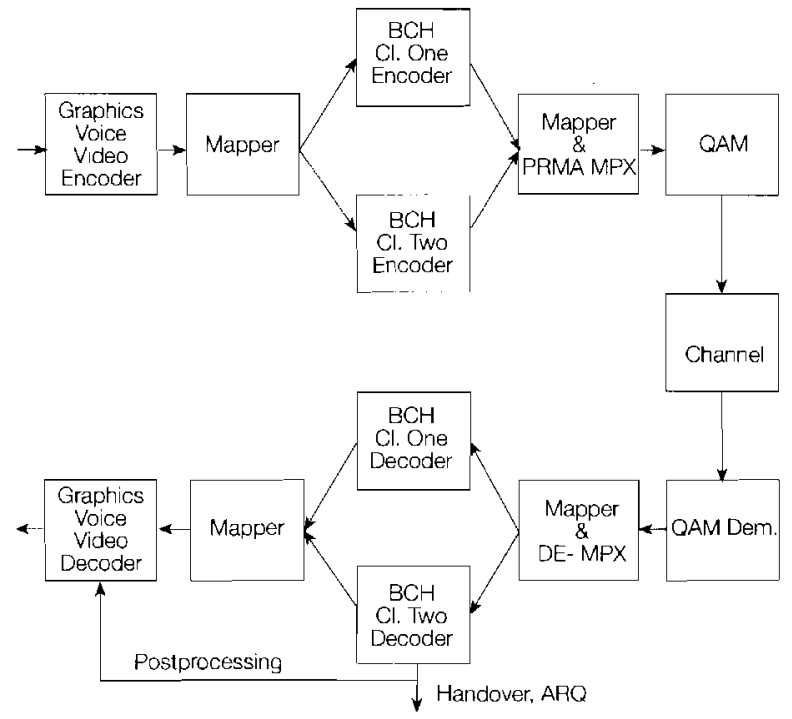

Fig. 1 - System's schematic.

Class Two source bits are fed to the Packet Reservation Multiple Access (PRMA) [13] Multiplexer (MPX) and queued for transmission to the Base Station (BS). Depending on the channel conditions, Pilot Symbol Assisted (PSA) 4-level or 16-level Quadrature Amplitude Modulation (QAM) [13] is invoked, allowing for the system to increase the number of bits per symbol within the same bandwidth and hence to improve the voice, video or graphics communications quality under benign channel conditions [9]. The receiver carries out the inverse functions and recovers the transmitted information. Should the communications quality unacceptably degrade, then the error detection capability of the stronger $\mathrm{BCH}$ code can be used to initiate a handover or Automatic Repeat Request (ARQ).

Specific issues of low-rate voice coding are considered for example in [10], while an intelligent multimode voice transceiver is proposed in reference [14]. In our current system a programmable-rate $8 / 4 \mathrm{kbit} / \mathrm{s}$ Code Excited Linear Predictive (CELP) speech codec is assumed [16] for the purposes of networking studies, but speech coding aspects are not considered here. A range of adaptive video source codecs and videophone transceivers have been proposed in references [15], which produce an arbitrarily programmable, but constant rate $8 / 4 \mathrm{kbit} / \mathrm{s}$ video sequence. Hence these codecs are ideally suited for videophony over conventional voice channels. Again, for our newtorking studies the 8 $\mathrm{kbit} / \mathrm{s}$ videophone scheme of reference [15] is assumed, but the discussion of specific videophony aspects is beyond the scope of this treatise (l). Having reviewed the system's architecture let us now concentrate on the graphical source coding issues.

(1) For further information concerning voice and video transmission issues the interested reader is referred to the WWW home-page hltp://www-mobile.ecs.soton.ac.uk.

\section{FIXED LENGTH DIfFERENTIAL CHAIN CODING}

In chain coding $(\mathrm{CC})$ a square-shaped coding ring is slid along the graphical trace from the current pixel, which is the origin of the legitimate motion vectors, in steps represented by the vectors portrayed in Fig. 2. The bold dots in the Figure represent the next legitimate pixels during the graphical trace's evolution. In principle the graphical trace can evolve to any of the surrounding eight pixels and hence a three-bit codeword is required for lossless coding. Differential chain coding [2, 4] (DCC) exploits that the most likely direction of stylus movement is a straight extension, with a diminishing chance of $180^{\circ}$ turns. This suggests that the coding efficiency can be improved using the principle of entropy coding by allocating shorter codewords to more likely transitions and longer ones to less likely transitions. This argument is supported by the histogram of the differential vectors of a range of graphical source signals, including English and Chinese handwriting, a Map and a technical Drawing, portrayed in Fig. 3, where vectors $0,+1$ and -1 are seen to have the highest relative frequency.

In this treatise we embarked on exploring the potential of a novel graphical coding scheme dispensing with the variable length coding principle of conventional DCC codecs, which we refer to therefore as fixed length differential chain coding (FL-DCC). FL-DCC was contrived in order to comply with the time-variant resolul-tionand/or bit rate constraints of intelligent third-generation adaptive multimode terminals, which can be re-configured under network control to satisfy the momentarily prevailing tele-traffic, robustness, quality, etc system requirements. In order to maintain lossless graphics quality under lightly loaded traffic conditions, the FL-DCC codec can operate at a rate of $b=3$ bits/vector, although it has a higher bit rate than DCC. However, since in voice and video coding typically perceptually unimpaired lossy quantisation is used, we embarked on exploring the potential of the re-configurable FL-DCC codec under $b<3$ low-rate, lossy conditions.

Based on our findings in Fig. 3 as regards to the relative frequencies of the various differential vectors, we

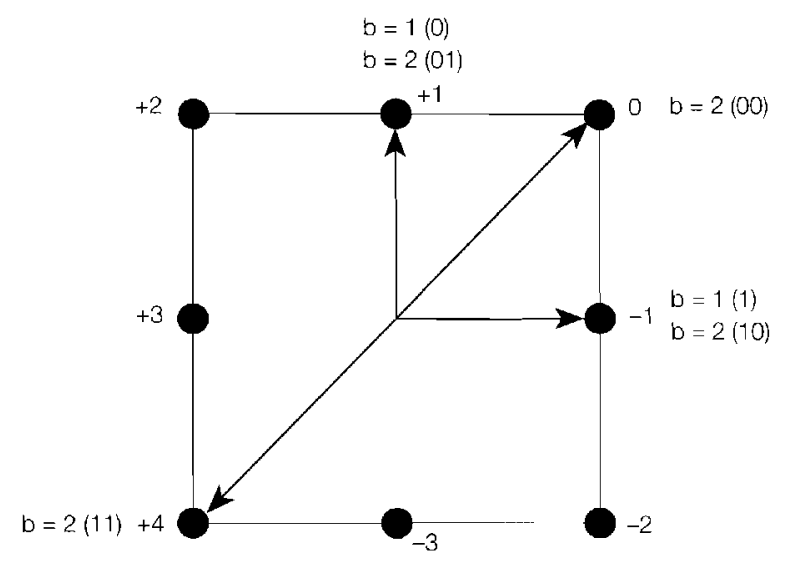

Fig. 2 - Coding ring. 




Fig. 3 - Relative frequency of differential vectors \%for a range of dynamographical source signals.

decided to evaluate the performance of the FL-DCC codec using the $b=1$ and $b=2$ bit/vector lossy schemes. As demonstrated by Fig. 2 , in the $b=2$ bit mode the transitions to pixels $-2,-3,+2,+3$ are illegitimate, while vectors $0,+1,-1$ and +4 are legitimate. In order to minimise the effects of transmission errors the Gray codes seen in Fig. 2 were assigned. It will be demonstrated that, due to the low probability of occurance of the illegitimate vectors, the associated subjective coding impairment is minor. Under degrading channel conditions or higher tele-traffic load the FL-DCC coding rate has to be reduced to $b=1$, in order to be able to invoke a less bandwidth efficient, but more robust modulation scheme or to generate less packets contending for transmission. In this case only vectors +1 and -1 of Fig. 2 are legitimate. The subjective effects of the associated zig-zag trace will be removed by the decoder, which can detect these characteristic patterns and replace them by a fitted straight line.

In general terms the size of the coding ring is given by $2 n \tau$, where $n=1,2,3 \ldots$ is referred to as the order of the ring and $\tau$ is a scaling parameter, characteristic of the pixel separation distance. Hence the ring shown in Fig. 2 is a first order one. The number of nodes in the ring is $M=8 n$.

The data syntax of the FL-DCC scheme is displayed in Fig. 4. The beginning of a trace can be marked by a typically 8 bit long pen-down (PD) code, while the end of trace by a pen-up (PU) code. In order to ensure that these codes are not emulated by the remaining data, if this would be incurred, bit stuffing must be invoked. We found however that in complexity and robustness terms using a 'vector counter' (VC) for signalling the trace-length to the decoder constituted a more attractive alternative for our system. The starting coordinates $X_{0}$, $Y_{0}$ of a trace are directly encoded using for example 10 and 9 bits in case of a video grahics array (VGA) resolution of $640 \times 480$ pixels.

The first vector displacement along the trace is encoded by the best fitting vector defined by the coding ring as the starting vector (SV). The coding ring is then translated along this starting vector to determine the next vector. A differential approach is used for the encoding of

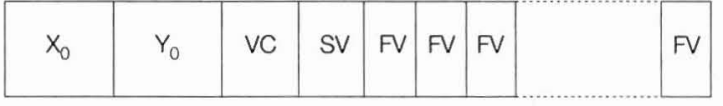

Fig. 4 - Coding syntax.

all the following vectors along the trace, in that the differences in direction between the present vector and its predecessor are calculated and these vector differences are mapped into a set of $2^{b}$ fixed length b-bit codewords, which we refer to as 'fixed vectors' (FV). We will show that the coding rate of the proposed FL-DCC scheme is lower for $b=2$ and $b=1$ than that of DCC.

When a curve is encoded by FL-DCC, it is sliced by the coding ring into small segments. Consider a sampled curve segment $s$. Let $v$ be the vector link produced by the coding ring. The coding rate of a chain code is defined $[1,3]$ in bits per unit length of the curve segment as

$r=\frac{E[b(s, v)]}{E\left[l_{n}(s)\right]}$

where $b(s, v)$ is the number of bits used to encode a vector link $v, l_{n}(s)$ is the length of the curve segment $s$, while $E(x)$ represents the expected value of a random variable $x$. It has been shown [4] that for the set of all curves, the product of a segment length $l_{n}(s)$ and the probability $p(\alpha)$ that this segment occurs with a direction alpha must be constant. Thus the expected curve segment length for a ring of order $n$ is given by [2]

$E\left[l_{n}(s)\right]=\int_{0}^{\pi / 4} \frac{8 \cdot n \cdot \tau}{\cos \alpha} \cdot p(\alpha) \mathrm{d} \alpha=\frac{\pi \cdot n \cdot \tau}{2 \cdot \sqrt{2}}$

Therefore, the theoretical coding rate of FL-DCC becomes:

$r=\frac{E[b(s, v)]}{\pi \cdot n \cdot \tau /(2 \cdot \sqrt{2})}$

The theoretical and experimental coding rates of the $b=1$ and $b=2$ FL-DCC schemes are shown in Table 1. 
Table 1 - Coding rate comparison

\begin{tabular}{|l|l|c|c|}
\hline & $\begin{array}{c}b=1 \\
\text { bit/vector }\end{array}$ & $\begin{array}{c}b=2 \\
\text { bit/vector }\end{array}$ & $\begin{array}{c}\text { DCC } \\
\text { bit/vector }\end{array}$ \\
\hline English script & 0.8535 & 1.7271 & 2.0216 \\
\hline Chinese script & 0.8532 & 1.7554 & 2.0403 \\
\hline Map & 0.8536 & 1.7365 & 2.0396 \\
\hline Drawing & 0.8541 & 1.7911 & 1.9437 \\
\hline Theoretical & 0.9 & 1.80 & 2.03 \\
\hline
\end{tabular}

In the next Section we endeavour to improve the robustness of the FL-DCC arrangement introduced, before transmission issues are considered.

\section{Robust FL-DCC}

It is well understood that the objective and subjective effects of transmission errors is different in memoryless coding schemes, such as for example Pulse Code Modulation (PCM), and in predictive schemes, such as Differential PCM (DPCM) [7, 8]. Our proposed FL-DCC scheme is to a certain extent related to DPCM, since it uses the previous vector as a prediction of the current one. This similarity prompted us to invoke a hybrid PCMDPCM-like technique suggested by Bull $[7,8]$ in order to improve the robustness of the FL-DCC codec using the schematic of Fig. 5.

Bull's hybrid scheme [7] is adopted in our FL-DCC scheme as follows.As it is demonstrated by the Figure, the $b$ bit FL-DCC codeword $u(n)$ is modified before transmission by modulo- $2^{b}$ adding the quantity $a(n)$, which is derived from the locally decoded graphical signal $y^{\prime}(n)$ by delaying it and taking the $b$ Most Significant Bits (MSBs) of it, which is carried out by the quantiser $Q^{*}$. Note that the modulo- $2^{b}$ addition truncates the result of the operation, which introduces some grade of arithmetic inaccuracy in comparison to an arithmetic treating overflows, but ensures that the transmitted codeword is still represented by $b$ bits. Under error-free conditions the encoder and decoder are perfectly "aligned" with eachother, implying that $a(n)=a^{\prime}(n), u(n)=u^{\prime}(n)$ and $y^{\prime \prime}(n)=y^{\prime}(n)$, which naturally allows unimpaired graph-

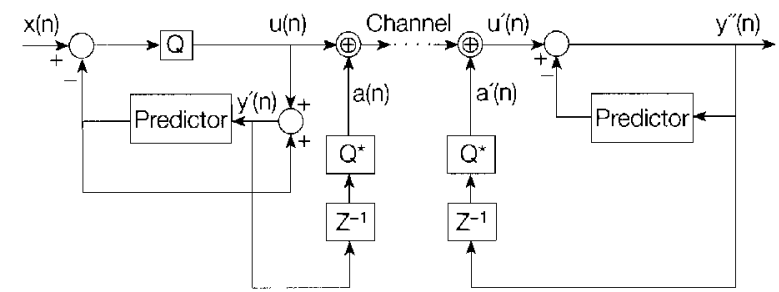

ical signal reconstruction, since the decoder carries out the inverse operations of the encoder.

In case of channel errors, however, the encoder and decoder become misaligned, but the proposed arrangement mitigates the propagation of transmission errors in the feed-back loop, as we will highlight in our forthcoming discourse. Let us assume that the decoded graphical signals at the encoder and decoder, namely $y^{\prime}(n)$ and $y^{\prime \prime}(n)$, respectively, now differ at the sampling instant $n$ due to transmission errors by the nonzero error quantity

$e(n)=y^{\prime}(n)-y^{\prime \prime}(n)$

Since the quantities $a(n)$ and $a^{\prime}(n)$ are derived from $y^{\prime}(n)$ and $y^{\prime \prime}(n)$ by coarse quantisation using $Q^{*}$, the difference $a(n+1)-a^{\prime}(n+1)$ at instant $n+1$ consitutes a good measure of the error's influence at instant $n+1$, because the decoded signals are delayed by one sampling interval. Bull [7] showed that the errors' effect can be substantially mitigated at sampling instant $n+1$, which will be demonstrated below, for the sake of conceptual simplicity assuming infinite precision addition, rather than modulo- $2^{b}$ truncation. From eq. (4) we have:

$y^{\prime \prime}(n)=y^{\prime}(\mathrm{n})-e(n)$

and by the help of the decoder's inner predictor loop of Fig. 5, which is constituted by a one-sample delay, we can proceed to sampling instant $n+1$ to arrive at:

$y^{\prime \prime}(n+1)=u^{\prime}(n+1)+y^{\prime \prime}(n)$

and substituting $y^{\prime \prime}(n)$ from eq. (5) into eq. (6):

$y^{\prime \prime}(n+1)=u^{\prime}(n+1)+y^{\prime}(n)-e(n)$

We argued above that the difference $a(n+1)-a^{\prime}(n+$ 1 ) is a good measure of the error's influence at instant $n+$ 1 , hence $u^{\prime}(n+1)$ in eq. (7) can be written as:

$u^{\prime}(n+1) \approx u(n+1)+a(n+1)-a^{\prime}(n+1)$

and substituting eq. (8) back in eq. (7), while exploiting that $a(n+1)-a^{\prime}(n+1) \approx e(n)$ leads to:

$$
\begin{aligned}
& y^{\prime \prime}(n+1)=u(n+1)+a(n+1)-a^{\prime}(n+1)+ \\
& y^{\prime}(n)-e(n) \approx u(n+1)+y^{\prime}(n)=y^{\prime}(n+1)
\end{aligned}
$$

which implies that the error effects are approximately cancelled at instant $n+1$. Above we have assumed an infinite precision arithmetic, rather than modulo- $2^{b}$ arithmetic, but in practice the accuracy of the error cancellation is degraded by the modulo- $2^{b}$ truncation. Having characterised the proposed FL-DCC codec let us now focus our attention briefly on the associated transmission issues. 


\subsection{Error control}

Trellis coded modulation (TCM) or block coded modulation (BCM) have been proposed in the literature in order to reduce the required channel $S N R[18,19]$, while in [20] source-matched joint source/channel coding and modulation providing un-equal error protection was introduced. This was achieved by exploiting that the so-called maximum minimum distance square-shaped 16-QAM constellation exhibits a higher- and a lower-integrity subchannel, naturally amenable to accommodate higher- and lower-sensitivity bits, respectively. This property was analysed in depth in [13]. In this treatise we will follow a similar design philosophy in order to achieve best system performance over fading channels [9].

Convolutional codes achieve their highest coding gain when relying on soft-decision decoding [11]. They do not retain reliable error detection capabilities and hence they are often combined with an external error detecting block code, as in the GSM system [12]. In contrast, high minimum-distance block codes have a highly reliable error detection capability [11], which is a useful feature in terms of monitoring the channel's quality in order to activate Automatic Repeat Requests (ARQ), handovers or error concealment. Based on these arguments in our system we favoured binary Bose-ChaudhuriHocquenghem (BCH) codes [11], which conveniently curtail channel error propagation across consecutive graphical traces. Although the soft-decision based trellis decoding of short block codes is potentially feasible in implementational complexity terms, the additional performance gain does not fully justify the complexity investment. This issue was illustrated in the context of the short $\operatorname{BCH}(15,11,1)$ code in [11]. When a higher complexity and interleaving delay are acceptable, the employment of the iterativelydecoded so-called turbo codes proposed by Berrou [21] and refined for example by Hagenauer et al $|22|$ is more beneficial in performance terms, achieving a performance close to the predictions of Shannonian information theory. For the sake of low complexity in this treatise we opted for conventional Berlekamp-Massey-Forney hard-decoding [11].

The system's performance can be further improved using Automatic Repeat Request (ARQ) techniques, if the associated increased delay is acceptable and there are un-used time slots available. Due to their delay and the additional requirement for a feed-back channel for message acknowledgement ARQ techniques have not been employed in the past in interactive speech or video communications. In our Packet Reservation Multiple Access (PRMA) system however there exists a full duplex control link between the BS and PS, which can be used for acknowledgements and the short PRMA frame length and microcellular propagation delay guarantee a low packet delay, therefore the employment of ARQ becomes realistic.
In second-generation mobile radio systems, such as for example the Pan-European GSM system [12] or the Digital European Cordless Telecommunications (DECT) scheme, non-linearly amplified constant envelope partial response modulation techniques have often found favour. Specifically, Gaussian Minimum Shift Keying (GMSK) has been advocated due to its spectral compactness and innate robustness against fading and amplifier non-linearities. However, in recent years rescarchers have turned their attention towards the concept of the Intelligent Multimode Terminal (IMT) [6], which by optimally reconfiguring itself in order to satisfy dynamically changing system optimisation criteria, achieve a better overall system performance. Spectral economy is further improved using micro- and pico-cellular structures, which exhibit friendly propagation properties and hence multilevel modulation schemes can be invoked in order to further increase the teletraffic delivered, as in the American IS-54 and the Japanese Digital Cellular (JDC) system.

A further advantage of linearly amplified multi-level modems is that they conveniently lend themselves to channel-quality or teletraffic-induced signal constellation re-configuration, an issue treated in depth for example in Chapter 13 of [13]. In order to complement the proposed multi-rate FL-DCC graphical source codec we designed a re-configurable modem. The most robust but least bandwidth efficient 4-level Quadrature Amplitude Modulation (4QAM) [13] mode can be used in outdoors scenarios in conjunction with the $b=1$ mode of the FL-DCC codec. The less robust but more bandwidth efficient 16QAM mode may be invoked in friendly indoors cells in order to accommodate the $b=2$ mode of operation of the FLDCC codec. When the channel conditions are extremely favourable, the modem can also be configured as a 64QAM scheme, in which case it can deliver $b=3$ bits per FL-DCC vector, allowing lossless coding. When using coherent PSAM [23], typically $3 \mathrm{~dB}$ lower channel signal-to-noise ratio $(S N R)$ is sufficient over AWGN channels than in case of non-coherently detected lowercomplexity StQAM modems [13], hence here we favour diversity-aided rectangular PSAM schemes [13, 23].

In our former work we found |13| that the Graycoded maximum-minimum distance rectangular 16QAM constellation exhibits two independent 2 bit subchannels having different bit error rates $(B E R)$, which naturally lend themselves to un-equal protection coded modulation. This issue was described in depth in Chapter 5 of [13]. Quantitatively, the BER of the lower quality class $2(C 2)$ subchannel was found a factor $2-3$ times higher than that of the higher integrity class 1 (C1) subchannel. Hence the more vulnerable FL-DCC coded bits will be transmitted via the $\mathrm{C}$ 1 subchannel, while the more robust bits over the $C 2$ subchannel. While previously proposed PSAM schemes used either a low-pass interpolation filter [24] or an approximately Gaussian filter [25], Cavers deployed a time-variant 
Wiener filter to minimise the channel estimation error variance. However, this computationally demanding technique has a similar performance to the conventional sinc-interpolator. In our quest for the best interpolator we found [17] that a simple polynomial interpolator had a similar performance to the above mentioned more complex schemes in terms of both mean squared estimation error as well as $B E R$ performance.

\subsection{Packetisation aspects}

In order to determine the desirable length of the transmission packets, in Fig. 6 we evaluated the histogram of the average trace length, which exhibited a very long low-probability tail. This tail probability was represented by the bars at an encoded trace-length of 200 bits in the Figure. Observe furthermore that, as expected, the highest concentration of short traces was recorded for $b=1$, which was followed by $b=2$ and the DCC mode of operation. However, most traces generated less than a few hundred bits, even when $b=2$ was used. In order to be able to use a fixed packet length, while maintaining robustness against channel errors and curtailing transmission error propagation across packets and/or traces, we decided to tailor the number of bits per trace to the packet length of 222 bits. If a longer trace was encountered, it was forcidly truncated to this length and the next packet started with a new 'artificial' pen-down code. If, however, a shorter trace was encountered, a second trace was also fitted in to the current packet and eventually truncated to the required length for transmission. Unfortunately, the additional forcidly included VC code and the $X_{0}, Y_{0}$ coordinates portrayed in Fig. 4 increased the number of bits generat- ed but mitigated the error propagation effects. The proportion of the bit rate increase evaluated in terms of $\%$ for various packet lenghts in case of the FL-DCC $b=1$ scheme is portrayed in Fig. 7.

Recall that the C1 16QAM subchannel had a factor 2-3 times better $B E R$ than the $C 2$ subchannel. This ratio would remain approximately the same if we were to use the same FEC code in both subchannels, but the $\mathrm{C} 2$ BER would remain excessively high for the transmission of the starting vectors (SV) and fixed-length vectors (FV) of Fig. 4. The $\mathrm{BCH}(255,131,18)$ and $\mathrm{BCH}(255,91,25)$ codes were found to ensure the required balance between the more and less robust FL-DCC bits, when employed in the $C 1$ and $C 2$ 16QAM subchannels, respectively. In other words, employing the more powerful $\operatorname{BCH}(255,91,25)$ codec in the higher-BER C2 16QAM subchannel reduced the integrity difference between the subchannels and ensured the required source-sensitivity matched unequal error protection. The $2 \times 255=510$ BCH-coded bits constitute 1284 bit 16QAM symbols. After adding 14 pilot symbols according to a pilot spacing of 10 and concatenating 4 ramp symbols for smooth power amplifier ramping in order to minimise the out-ofband emissions the resulting 146-symbol packets are queued for transmission to the BS. The same packet format can be used for the voice/video packets. Both the voice and the video codecs generate in their $8 \mathrm{kbit} / \mathrm{s}$ mode of operation $160 \mathrm{bits} / 20 \mathrm{~ms}$ frame and hence the 222 bit packet can accommodate a 62 bit signalling and control header in each 146-symbol packet. The corresponding single-user voice/video signalling rate becomes 146 symbols $/ 20 \mathrm{~ms}=7.3 \mathrm{kBd}$. Due to their significantly lower rates and higher delay tolerance graphical users assemble their 146-symbol transmission packets over a

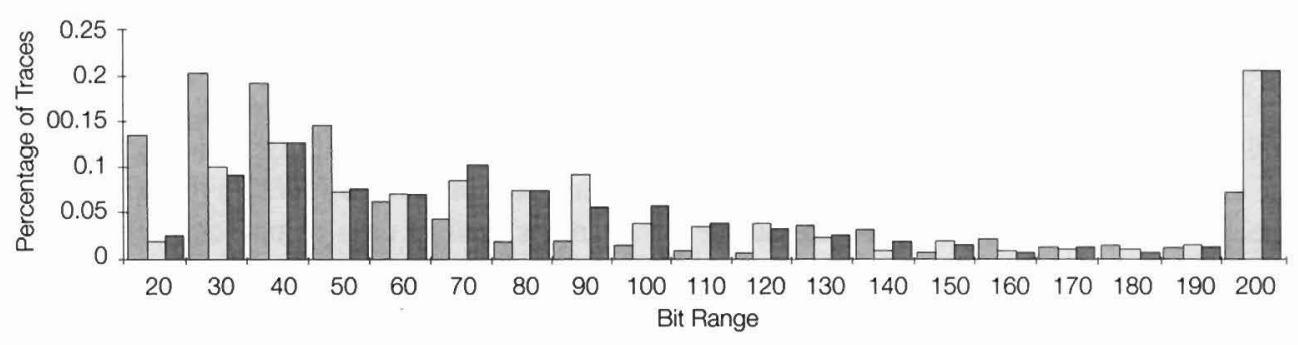

$\square$ FL-DCC ( $(\mathrm{b}=1) \quad \square$ FL-DCC $(\mathrm{b}=2) \quad \square \mathrm{DCC}(\mathrm{M}=8)$

Fig. 6 - Histogram of the average trace length.

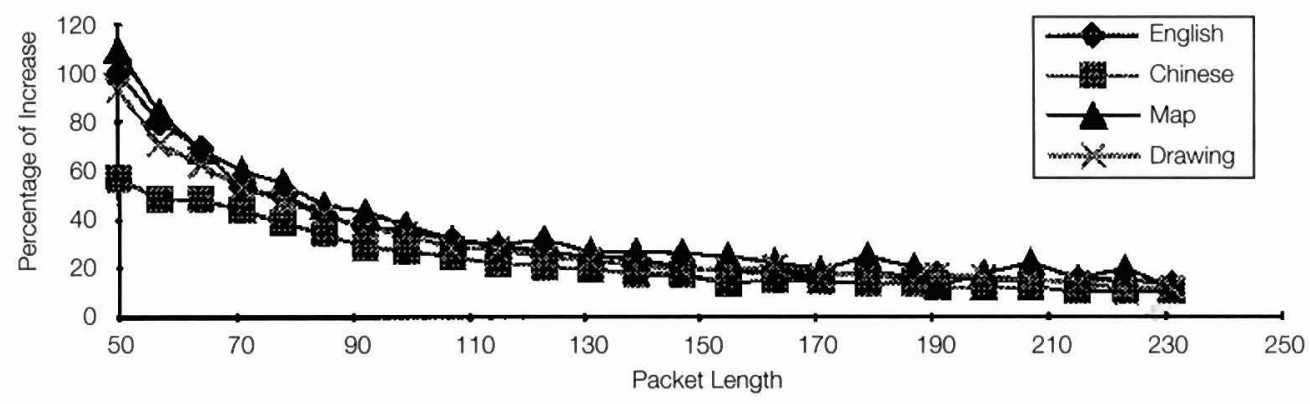

Fig. 7 - Proportion of bit rate increase due to fixed-length trace termination versus packet length for the FL-DCC $b=1$ scheme. 

tion. Apart from carrying out this function the PRMA multiplexer facilitates a more efficient exploitation of the transmission medium by allocating packets on a flexible demand basis to the graphics, voice and video users. Since our video codec [15] delivers a constantrate $8 \mathrm{kbit} / \mathrm{s}$ stream, the video information is conveyed using regularly-spaced TDMA-packets. Hence no video delay and packet dropping is inflicted and no buffer memory is required. In contrast, human speech has bursty statistics and the proportion of active speech spurts is around [28], which can be exploited to support a number of additional speech users [26] or to accommodate a mixture of speech and data users [27].

The voice activity detector [12] queues speech packets for transmission to the BS and if the Portable Station (PS) gets permission to transmit, it reserves the time-slot for the duration of the current active speech spurt. If a passive speech spurt is detected, the time-slot is surrendered and can be reserved by other speech, data or video users, who are becoming active. If, however, due to packet collisions a speech user cannot get a reservation within the maximum tolerable delay of about $30 \mathrm{~ms}$, the validity of the current speech packet expires, since new speech packets may be awaiting transmission. Hence this initial packet must be dropped but the dropping probability must be kept below $P_{\text {drop }}=1 \%$ [26] in order to minimise the speech degradation. Fortunately this initial spurt clipping is hardly perceivable.

In contrast, graphical data packets cannot be dropped, but tolerate longer delays and can be allocated to slots, which are not reserved by speech users in the present frame. In our experiments all but one graphical data users were obeying a negative exponential packet generation model, while one user transmitted graphical traces generated by a writing tablet.

A less than unity permission probability either allowed or disabled permission to contend during any particular slot for a reservation within the current PRMA frame. Wong and Goodman noted [27] that it is advantageous to control data packet contentions on the basis of the fullness of the contention buffer. Specifically, contentions are disabled, until a certain number of packets awaits transmission, which reduces the probability of potential packet collisions due to the frequent transmission of short graphical data bursts. While speech communications stability can be defined as a dropping probability $P_{\text {drop }}<1 \%$, data transmission stability is specified in terms of maximum graphical data delay or buffer requirement. The PRMA packet multiplexer is depicted in Fig. 8, where we indicated in the Figure the required permission probability $P$ used by the various speech, data and video users in order to satisfy their corresponding delay and packet dropping constraints, while maximising the multiplexer's throughput capacity. The key PRMA parameters, including these speech, video and data permission probabilities, are listed in Table 2 along with a range of other system features.

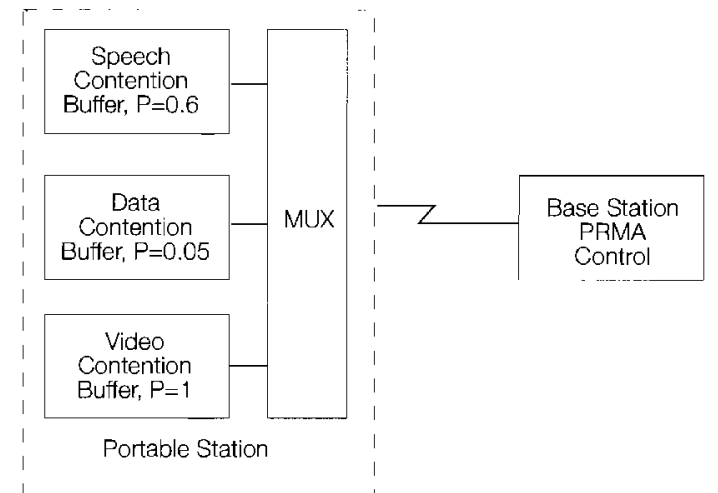

Fig. 8 - PRMA packet multiplexer.

\section{RESULTS AND DISCUSSION}

The previously described components of the system were simulated and amalgamated. The range of simulation conditions will be specified throughout the forthcoming three subsection, describing the FL-DCC graphical codec performance, the PRMA multiplexer's performance and the overall system performance, respectively.

\subsection{FL-DCC graphical codec performance}

The performance of the proposed FL-DCC schemes was evaluated for a range of dynamographical source signals, including an English script, a Chinese script, a drawing and a map using a coding ring of $M=8$. Table 1 shows the associated coding rates produced by FL-DCC for $b=1$ and $b=2$ as well as by DCC along with the corresponding theoretical coding rates. Both FL-DCC schemes achieve a lower coding rate than DCC. The corresponding subjective quality is portrayed in Fig. 9 for two of the input signals previously used in Table1. Observe that for $b=2$ no subjective degradation can be seen and the degradation associated with $b=1$ is also fairly low. This is due to the fact that the typical fuzzy granular error patterns inflicted by the $b=1$ FL-DCC scheme, when a straight line section is approximated by a zig-zag pattern, can be detected and smoothed by the decoder.

\subsection{Multimedia PRMA performance}

As seen in Table 2, the PRMA channel rate was 133 $\mathrm{kBd}$, and $181.11 \mathrm{~ms}$ slots can be created in a $20 \mathrm{~ms}$ frame, which can accommodate $7.3 \mathrm{kBd}$ source streams constituted by the pair of $\mathrm{BCH}(255,131,18)$ and $\mathrm{BCH}$ $(255,91,25) C 1$ and $C 2$ packets in the 16QAM mode. We dedicated nine time slots for video users on a TDMA-basis, who had a unity permission probability to contend, since our video codec generated a continuous, constant rate output stream and the delay had to be minimised. However, if less than nine multimedia users are present at any moment, the dedicated TDMA video slots may be offered for contention to voice and graphics data users, in order to optimally exploit the system's 

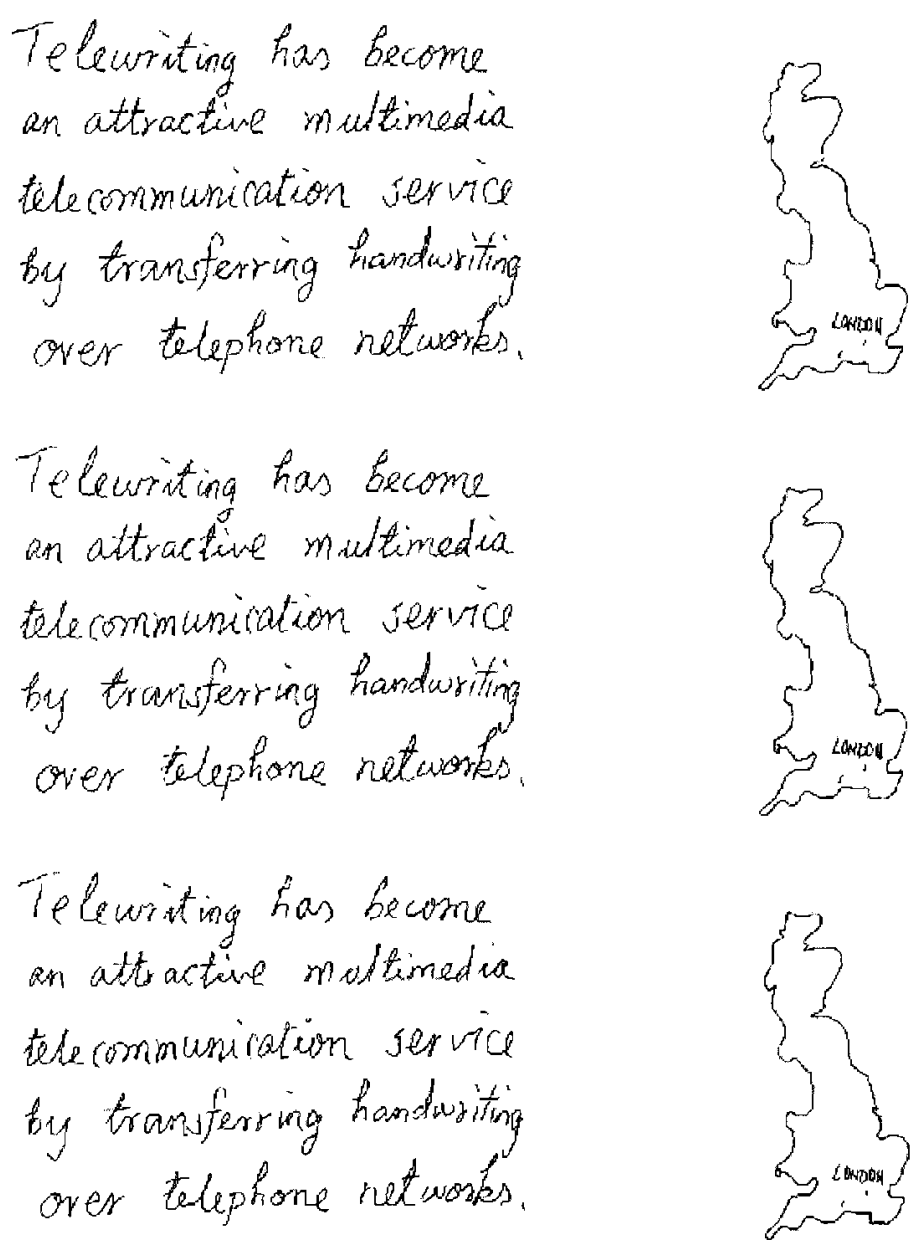

Fig. 9 - Decoded information for FL-DCC with $b=1$ (bottom), $b=2$ (centre) and DCC (top).

traffic throughput capacity. This would have the disadvantage that if suddenly a speech user wanted to activate the video mode, he/she would have to wait for a TDMA-slot to become available.

Having allocated the TDMA video-slots, the remaining nine slots were offered to mixed speech and graphical data users. We found that in this scenario the optimum speech and graphical data permission probabilities were 0.6 and 0.05 , respectively. The significantly lower data permission probability ensures that the delay-sensitive voice packets are not colliding with and delayed by agressively contending graphical data packets, which can more readily tolerate slight delays than interactive speech packets. These delay issues will be portrayed in Fig. 10. The total traffic carrying capacity of the PRMA multiplexer can be expressed in a variety of ways in terms of the number of speech, video and data users supported, which is typically related to the system stability limit. In our system stability was defined for speech users as a packet dropping probability of $P_{\text {drop }}<1 \%$, and for graphical data users as a maximum data buffer length of 100 packets, each delivering a 146 symbol packet. The total maximum storage required was then $100 \times 146 \times 4 / 8=7.3$ kbytes. The PRMA multiplexer's efficiency can be quantified in terms of the slot occupancy, which ideally should be as close to $100 \%$ as possible, but this requirement contradicts to maintaining stability. In other words, the slot occupancy can be only increased at the cost of increased speech packet dropping probability or data packet delay and buffer length. In order to limit the variety of traffic loading scenarios under which the system's capacity is studied, similarly to the number of videophone users, we stipulated the number of graphical data users as nine. This assumption follows the argument that the penetration of new video and graphical services is expected to be lower than that of the more established voice services.

The system's traffic loading was varied by adjusting the data transmission rate between 0.5 and $4 \mathrm{kbit} / \mathrm{s}$ in steps of $0.5 \mathrm{kbit} / \mathrm{s}$ using negative exponential packet arrival distribution and by setting the number of speech usersto $9,12,13$ and 14, who were generating speech spurts according to Brady's model [28]. Again, following Wong and Goodman [27], the minimum number of graphical data packets in the contention buffer to enable data contention was set to two, which slightly increased the mean data delay, but significantly reduced the chance of packet collision and dropping, while reducing the speech packet delay. The corresponding results are displayed in Figs. 10 - 12 for the mean data delay, maximum buffer size and slot occupancy, respectively, which were derived for a 
Data Delay (ms)

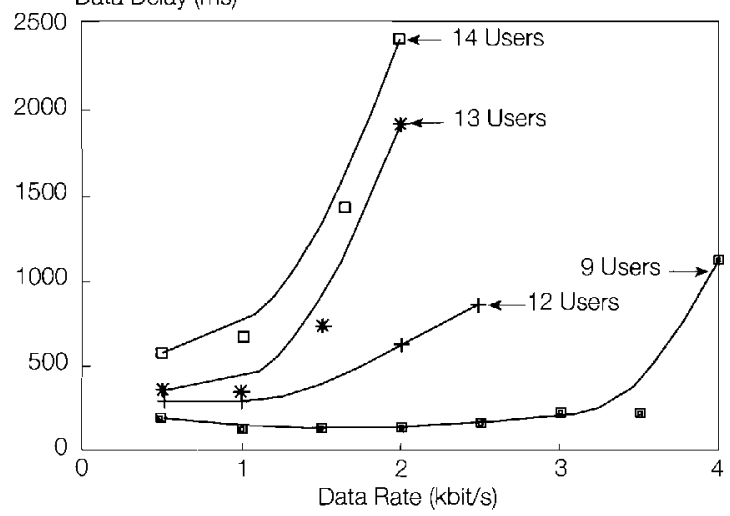

Fig. 10 - Graphical data buffer size versus data rate for various number of speech users using 16QAM.

200 s duration communications session, transmitting $10^{4}$ PRMA frames. As expected, the minimum mean data delay and required buffer size are maintained under lightly loaded tele-traffic conditions. Observe from these Figures that when supporting nine $8 \mathrm{kbit} / \mathrm{s}$ video and nine $0.5 \mathrm{kbit} / \mathrm{s}$ graphical data users as well as a maximum of $148 \mathrm{kbiu} / \mathrm{s}$ speech users, the mean data delay is less than about $600 \mathrm{~ms}$ and the required buffer size is below about 30 packets. Explicitly, due to speech burstyness and with the advent of voice activity detection and PRMA, the system can support up to 5 additional speech users with a dropping probability around $1 \%$ plus nine $1 \mathrm{kbit} / \mathrm{s}$ graphical users. This corresponds to a system capacity improvement of $5 \times 8+9 \times 1=49 \mathrm{kbit} / \mathrm{s}$, or a relative capacity increase of $49 /(9 \times 8)=68 \%$ over TDMA.

Upon interpreting Figs. 10 - 12 from a different angle, if only 9 simultaneous voice communications are conducted along with the previously stipulated 9 video and data sessions, the packet dropping probability is virtually zero and both the data delay and the buffer size curves are rather flat up to a maximum data rate of about $3.5 \mathrm{kbit} / \mathrm{s}$. At a rate of $3.5 \mathrm{kbit} / \mathrm{s}$ the stipulated graphical data stability limit of a buffer size of 100 packets is reached. The mean data delay becomes about $200 \mathrm{~ms}$. The data delay and buffer size curves of Figs. 10 and 11 become more steep, if more than 9 speech users must be accommodat-

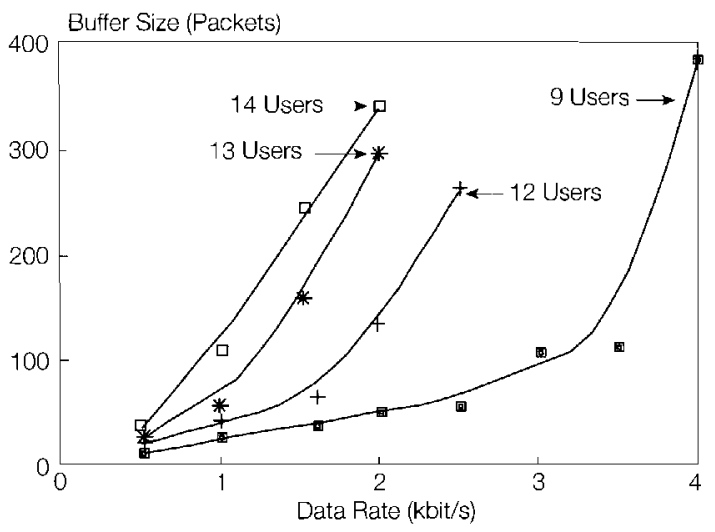

Fig. 11 - Graphical data delay versus data rate for various number of speech users using 16QAM.

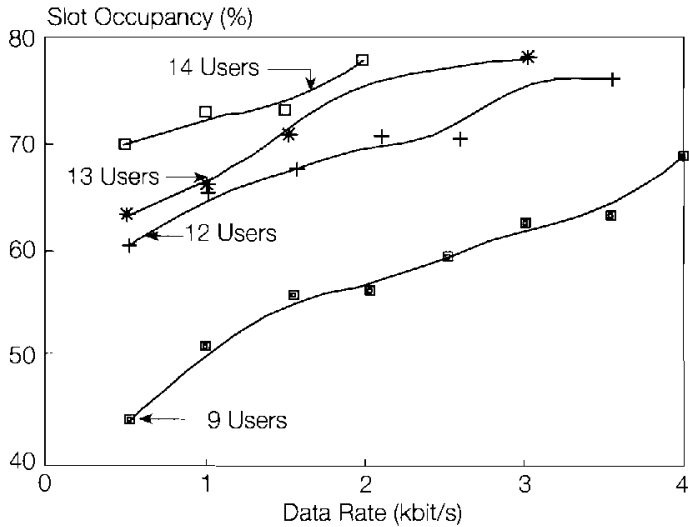

Fig. 12 - Slot occupancy versus data rate performance for various number of speech users using 16QAM.

ed, since the interactive speech users enjoy a higher contention probability. Note in Fig. 12 that the slot occupancy defined as the proportion of slots occupied by packets is below $80 \%$ in all the above stable scenarios. Again, apart from its multiplexing function, the benefit of PRMA was that due to speech burstyness we could provide an extra $3.5 \mathrm{kbit} / \mathrm{s}$ data channel for each of the nine speech/video users, which was equivalent to a channel capacity gain of $9 \times 3.5=31.5 \mathrm{kbit} / \mathrm{s}$. Relating this gain to the speech source rate of $9 \times 8=72 \mathrm{kbit} / \mathrm{s}$ a relative channel capacity gain of $31.5 / 72 \approx 44 \%$ is achieved. This is lower than the capacity gain of the more heavily loaded 14-user scenario, where the higher traffic loading exhibited itself in an increased packet dropping probability of about $1 \%$. These findings are also reflected by the slot occupancy curves of Fig. 12. Let us now focus our attention on the robustness aspects of the proposed graphical transceiver, when used over various wireless channels.

\subsection{Robustness issues}

Our experimental channel conditions were based on a worst-case Rayleigh-fading scenario using a propagation frequency of $1.9 \mathrm{GHz}$, signalling rate of $133 \mathrm{kBd}$ and vehicular speed of $30 \mathrm{mph}$. For reasons of space economy no modem $B E R$ versus channel SNR curves are plotted here, instead the overall system robustness will be characterised in Figs. 13 - 16. The graphical representation quality was evaluated in terms of both the mean squared error (mse) and the Peak Signal to Noise Ratio (PSNR). In analogy to the PSNR in image processing, the graphical PSNR was defined as the ratio of the maximum possible spatial deviation 'energy' to the coding error 'energy'. When using a resolution of $640 \times$ 480 pixels, the maximum spatial deviation energy is $640^{2}+4802=640000$, corresponding to a maximum diagonal spatial deviation of 800 pixels. The lossy cod ing energy was measured as the mean squared value of the pixel-to-pixel spatial distance between the original graphical input and the FL-DCC graphical output. For perfect channel conditions the $b=1$ and $b=2$ FL-DCC codec had PSNR values of 49.47 and $59.47 \mathrm{~dB}$, respec- 


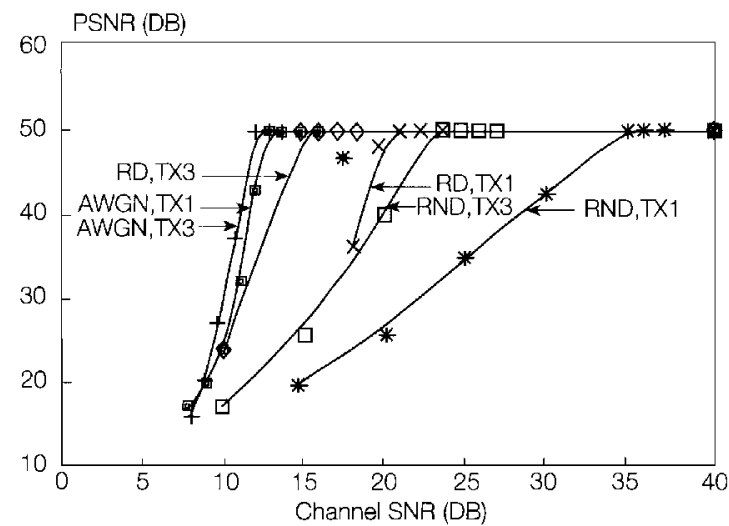

Fig. 13 - Graphical PSNR versus channel $S N R$ performance of the $b=$ 1 bit FL-DCC/16QAM mode of operation over various channels.

tively. As we showed in Fig. 9, the subjective effects of a $10 \mathrm{~dB}$ PSNR degradation due to using $b=1$ instead of $b=2$ are not severe in terms of readability.

The system's robustness was characterised in Figs. 13 16 upon transmitting the handwriting sequence of Fig. 9 ten times, in order to ensure the statistical soundness of the graphical quality investigations. The best-case propagation scenario was encountering the stationary Additive White Gaussian Noise (AWGN) channel. The more bandwidth-efficient but less robust 16QAM mode of operation is characterised by Fig. 13. Observe that transmissions over Rayleigh channels with diversity $(\mathrm{RD})$ and with no diversity (RND) are portrayed using both one (TX1) and three (TX3) transmission attempts, in order to improve the system's robustness. Two-branch selection diversity using two independent Rayleigh channels was studied in conjunction with various selection criteria and we found that using the channel with the minimum phase shift between pilots slightly outperformed the maximum energy criterion. Similarly, over AWGN channels one or three transmission attempts were invoked.

As seen in Figs. 13 and 14 in case of the 16QAM mode, over AWGN channels the required channel SNR for unimpaired graphical communications is around 11 $\mathrm{dB}$ with $\mathrm{ARQ}$ and $12 \mathrm{~dB}$ without $\mathrm{ARQ}$. This marginal improvement is attributable to the fact that the AWGN

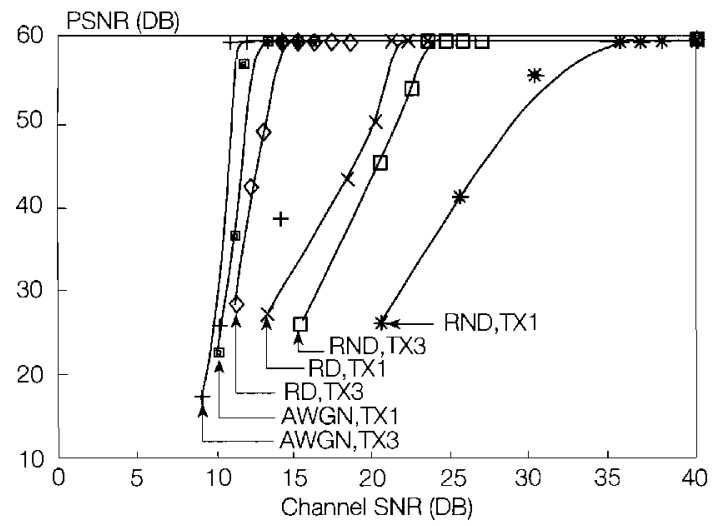

Fig. 14 - Graphical PSNR versus channel $S N R$ performance of the $b=$ 2 bit FL-DCC/16QAM mode of operation over various channels.



Fig. 15 - Graphical PSNR versus channel $S N R$ performance of the $b=$ 1 bit FL-DCC/4QAM mode of operation over various channels.

channel exhibits always a fairly constant bit error rate $(B E R)$ and hence during re-transmission attempts the chances of successful transmissions are only marginally improved. In contrast, over Rayleigh channels the received signal has a high probability of emerging from a deep fade by the time the packet is re-transmitted. This is the reason for the significantly improved robustness of the ARQ-assisted Rayleigh scenarios of Figs. 13 and 14. Specifically, with diversity the ARQ attempts reduced the required channel $S N R$ by about $5 \mathrm{~dB}$ to around $15 \mathrm{~dB}$, while without diversity an even higher 10-12 dB ARQ gain is experienced. When using the $b=$ 2 bit FL-DCC scheme, Fig, 14 shows that the error-free PSNR was increased to nearly $60 \mathrm{~dB}$, while the system's robustness against channel errors and the associated "corner $S N R$ " values remained unchanged.

Fig. 15 demonstrates that when the more robust 4QAM mode was invoked, over AWGN channels $S N R$ values of 5 and $8 \mathrm{~dB}$ were necessitated by the ARQaided TX3 scheme and the non-ARQ assisted TX1 systems, respectively. The diversity-assisted RD, TX3 and RD, TX1 systems required a minimum SNR of about 10 and $17 \mathrm{~dB}$ for unimpaired graphical communications, which had to be increased to 20 and $27 \mathrm{~dB}$ without diversity. Clearly, both the ARQ- and diversityassistance played a crucial role in terms of improving

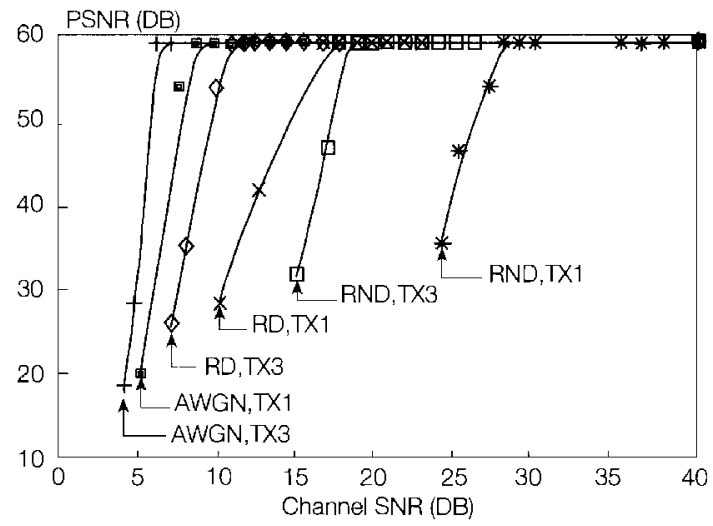

Fig. 16 - Graphical PSNR versus channel $S N R$ performance of the $b=$ 2 bit FL-DCC/4QAM mode of operation over various channels. 
channels, respectively. The system's robustness was improved and its tele-traffic capacity dropped, when 4QAM was invoked. Further key system features are summarised in Table 2 . Our future work is targeted at studying various adaptive modem reconfiguration algorithms and improving the bit rate, resolution and robustness balance of the system.

\section{Acknowledgement}

The financial support of the EPSRC, UK in the framework of the research contract GR/K74043 is gratefully acknowledged.

Manuscript received on January 24, 1996.

\section{REFERENCES}

[1] D. L. Neuhoff, K. G. Castor: A rate and distortion analysis of chain codes for line drawings. "IEEE Tran. Information Theory", Vol. IT-31, Jan. 1985, p. 53-68.

[2] K. Liu, R. Prasad: Performance analysis of differential chain coding. "European Transaction on Telecommunications and Related Technology", Vol. 3, Jul-Aug. I992, p. 323-330

[3] A. B. Johannessen, R. Prasad, N. B. J. Weyland, J. H. Bons: Coding efficiency of multiring differential chain coding. "IEE Proceedings", Part I, Vol. 139, April 1992, p. 224-232.

[4] R. Prasad, J. W. Vivien, J. H. Bons, J. C. Arnbak: Relative vector probabilities in differential chain coded line drawings. Proc. of IEEE Pacific Rim Conference on Comms., Computers and Signal Processing, Victoria, Canada, June 1989, p 138-142.

[5] R. Prasad, P. A. D. Spaargaren, J. H. Bons: Teletext reception in a mobile channel for a broadcast tele-information system. "IEEE Tr. on VT.", Vol. 42, No. 4, Nov. 1993, p 535-545.

[6] Advanced Communications Technologies and Services, Workplan, DCXIII-B-RA946043-WP, 1994.

[7] M. C. W. Van Bull: Hybrid D-PCM, a combination of PCM and DPCM. "IEEE Tr. on Comms.", Vol. Com-26, March 1978, p 362-368.

[8] N. S. Jayant, P. Noll: Digital coding of waveforms. PrenticeHaIl, 1984.

[9] D. Greenwood, L. Hanzo: Mobile radio channels. Chapter 2 In (R. Steele Ed.), Mobile Radio Communications, IEEE PressPentech Press, London, 1992, p 92-185.

[10] R. A. Salami, L. Hanzo, et al: Speech Coding. Chapter 3 In: (R. Steele Ed.), Mobile Radio Communications, IEEE PressPentech Press, London, 1992, p 186-346.
[11] K. H. H. Wong, L. Hanzo: Channel Coding. Chapter 4 In: (R. Steele Ed.), Mobile Radio Communications, IEEE PressPentech Press, London, 1992, p 347-489.

[12] L. Hanzo, J. Stefanov: The Pan-European Digital Cellular Mobile Radio System - known as GSM. Chapter 8 In: (R. Steele Ed.), Mobile Radio Communications, IEEE Press-Pentech Press, London, 1992, p 677-773.

[13] W. T. Wcbb, L. Hanzo: Modern quadrature amplitude modula tion: Principles and applications for fixed and wireless channels. "IEEE Press-Pentech Press", ISBN 0-7273-1701-6, 1994, p 557.

[14] L. Hanzo, J.P. Woodard: An intelligent multimode voice communications system for indoors communications. "IEEE Tr. on Veh. Technology", Vol. 44, No. 4, Nov. 1995, p 735-749.

[15] L. Hanzo, J. Streit: Adaptive low-rate wireless videophone schemes. "IEEE Tr. on Circuits, Systems and Video Tcchnology", Vol. 5, No. 4, Aug. 1995, p 305-319.

[16] J. P. Woodard: Digital coding of speech using code excited linear prediction. Phd. Thesis, Dept. of Elcctr. Univ, of Southampton, UK, Nov, 1995.

[17] J. Torrance, L. Hanzo: A comparative study of pilot symbo assissted modem schemes. Proc. of IEE RRAS'95 Conference, Bath, UK, Conf. Public. No. 415, 26-28 Sept. 1995, p 36-41.

[18] E. Biglieri, M. Luise: Coded modulation and bandwidth-efficient transmission. Proc, of the Fifth Tirrenia Intern. Workshop, 8-12 Sept. 1991, EIsevier, Netherlands, 1992.

[19] IEEE Communications Magazine, Special Issue on Coded Modulation, Vol 29, No 12, Dec. 1991.

[20] L.F. Wei: Coded modulation with unequal error protection. "IEEE Tr. on Comms.", Vol. 41, No. 10, Oct. 1993, p 1439-1450.

[21] C. Berrou, P. Thitimajshima: Near shannon limit error-correction coding and decoding: Turbo-codes. In Proceedings of the International Conference on Communications (ICC'93), (Geneva), May 1993, p. 1064-1070.

[22] J. Hagenauer, E. Offer, L. Papke: Iterative decoding of binary block and convolutional codes. "IEEE Transactions on Information Theory", Vol. 42, 1996, p. 429-445.

[23] J. K. Cavers: An analysis of pilot symbol assisted modulation for rayleigh fading channels. "IEEE Tr. on VT", Vol. 40, No 4, Nov. 1991, p 686-693.

[24] M. L. Moher, J. H. Lodge: TCMP - a modulation and coding strategy for rician fading channels. "IEEE J. Select. Areas Commun.", Vol.7, Dec. 1989, p. 1347-1355.

[25] S. Sampei, T. Sunaga: Rayleigh fading compensation method for 16-OAM in digital land mobile radio channels. Proc. IEEE Veh. Technol. Conf., San Francisco, CA, May 1989, p. 640-646.

[26] D. J. Goodman, S. X. Wei: Efficiency of packet reservation multiple access. "IEEE Transactions on Vehicular Technology", Vol. 40, No. 1, Febr. 1991, p. 170-176

[27] W. Wong, D. Goodman: A packet reservation access protocol for integrated speech and data transmission. "Proc. of the IEE, Part-I", Vol 139, No 6, Dec. 1992, p 607-613.

[28] P. T. Brady: A model for generating on-off speech patterns in two-way conversation. "Bell System Technical Journal", Vol. 48, No. 7, September, 1969, p. 2445-2472. 